A N N A L E S Annales de Bretagne et des Pays de l'Ouest

\title{
Lorient, ville portuaire. Une nouvelle histoire des origines à nos jours
}

\section{Bernard Michon}

\section{(2) OpenEdition}

1 Journals

Édition électronique

URL : https://journals.openedition.org/abpo/6602

DOI : $10.4000 / a b p o .6602$

ISSN : 2108-6443

Éditeur

Presses universitaires de Rennes

\section{Édition imprimée}

Date de publication : 10 décembre 2020

Pagination : 225-227

ISBN : 978-2-7535-8225-5

ISSN : 0399-0826

Référence électronique

Bernard Michon, "Lorient, ville portuaire. Une nouvelle histoire des origines à nos jours », Annales de Bretagne et des Pays de l'Ouest [En ligne], 127-4 | 2020, mis en ligne le 10 décembre 2020, consulté le 06 janvier 2023. URL : http://journals.openedition.org/abpo/6602 ; DOI : https://doi.org/10.4000/ abpo.6602 
cisément datés mais culturellement très intéressants, qu'il s'agisse du vol sacrilège de la croix d'or de l'église de Plouaret ou de la circulation en Bretagne et en Europe du motif du vieillard aveugle qui donne des conseils pour choisir la terre où bâtir sa maison.

C'est avant tout l'enquête policière qui intéresse Daniel Giraudon et qu'il nous livre pas à pas au cours d'une lecture passionnante, tant sur les chemins du Trégor où il a réalisé la majorité de ses enquêtes ethnographiques que dans les dépôts d'archives. On ne trouvera pas dans cet ouvrage une approche théorique sur l'usage des sources orales comme matière pour l'histoire. C'est par le cas pratique que l'auteur fait sa démonstration, en assumant une analyse qui touche parfois à l'érudition. Mais c'est ce souci de recherche pointilleuse qui permet de mettre à jour de façon convaincante la datation de nombreux faits divers ayant donné lieu à des gwerzioù, permettant ainsi d'augmenter significativement le nombre de datations de complaintes bretonnes des $\mathrm{XVII}^{\mathrm{e}}$ et XVIII ${ }^{\mathrm{e}}$ siècles par rapport au corpus déjà recensé. Certains regretteront peut-être une lecture très positiviste des traditions orales ('analyse se concentre avant tout sur la possibilité ou non de vérifier si les informations fournies par les chansons sont historiquement exactes) et l'absence d'une remise en perspective large de chaque cas au sein du répertoire plus vaste des complaintes en Bretagne ou ailleurs et en lien avec les travaux réalisés par d'autres chercheurs. Mais les compétences de l'ethnographe, la richesse et la connaissance des sources orales recueillies et mobilisées par le collecteur sont remarquables. Les conclusions et hypothèses proposées quant à l'interaction entre oral et écrit et l'indication des auteurs supposés des compositions permettront pour leur part d'alimenter les débats sur l'origine et la circulation des traditions orales.

Ces études ont pour la plupart, et dans des formats proches, déjà été publiées sous la forme d'articles éclatés - dont plusieurs dans les Annales de Bretagne et des Pays de l'Ouest - et il est dommage que les références aux premières publications n'aient pas été mentionnées dans l'ouvrage. Mais l'idée rassembler ces travaux en un seul volume est tout à fait judicieux : au-delà des comparaisons possibles entre les chansons ou de la mise à disposition auprès d'un large public d'articles d'abord publiés en breton (dans le cas de la gwerz sur la mort de Toussaint de Kerguézec en 1709), la juxtaposition des chapitres permet de mesurer à quel point l'ancrage des gwerzioù dans l'histoire locale est une donnée caractéristique de ce répertoire. L'ouvrage offre en outre une plus-value précieuse par rapport aux articles précédemment publiés à travers la riche iconographie en noir et blanc et en couleur qui accompagne chaque étude : les photos des interprètes et des lieux nommés dans les complaintes notamment, prises pour la plupart par l'auteur lui-même, sont très appréciables. Il ne manque que le son, et on espère donc que le prochain ouvrage de Daniel Giraudon rassemblera les textes, partitions et enregistrements sonores de son exceptionnelle collecte de chansons de tradition orale en Bretagne.

Éva GuILLOREL

LE BOUËDEC, Gérard et CÉRINO, Christophe, Lorient, ville portuaire. Une nouvelle histoire des origines à nos jours, préface de Norbert Métairie, Rennes, Presses universitaires de Rennes, Hors collection, 2017, 398 p.

En 1988, les éditions Privat publiaient une Histoire de Lorient sous la direction de Claude Nières, dans leur fameuse collection consacrée aux villes de France. 
Moins de trente ans plus tard, ce beau et solide volume permet de mesurer les progrès accomplis par la recherche scientifique sur ce sujet, notamment au sein de l'université de Bretagne Sud, fondée officiellement en 1995, dont la ville de Lorient accueille les formations en Lettres, Sciences Humaines et Sociales à l'intérieur de son bâtiment "Le Paquebot ". Dans sa préface, Gérard Le Bouëdec, professeur émérite d'histoire maritime à l'université de Bretagne Sud, présente d'ailleurs ce livre comme un " hommage " à ses étudiants de master et à ses doctorants. La bibliographie (p. 353-370), organisée autour de quatre entrées - Histoire du Pays de Lorient; Histoire du Morbihan; Histoire de la Bretagne; Histoire maritime nationale et internationale - rend compte de cette dynamique, en consacrant à chaque fois une rubrique fournie aux " Travaux universitaires ". Les publications des deux auteurs ne sont pas oubliées - pas moins de 120 titres pour Gérard Le Bouëdec et 30 pour Christophe Cérino, ingénieur de recherche à l'université de Bretagne Sud qui s'est spécialisé dans l'histoire de la base de sous-marins de Keroman après sa thèse consacrée à Belle-Île-en-Mer sous le règne de Louis XV - et attestent de leur investissement majeur pour une meilleure connaissance historique du Pays de Lorient en particulier, mais également de l'histoire maritime en général. Ils ont ensemble été à l'origine du Groupement d'intérêt scientifique (GIS) d'histoire maritime, créé en 2005, devenu depuis 2015 GIS d'Histoire et Sciences de la mer, qui fédère actuellement 36 établissements français et 35 étrangers. Au-delà de l'université, l'ouvrage doit également beaucoup aux ressources et aux productions des différentes institutions présentes sur le territoire, du Service historique de la Défense au musée de la Compagnie des Indes, en passant par les archives municipales de Lorient, sans oublier les différentes sociétés savantes locales. Les nombreuses illustrations qui agrémentent le volume en témoignent : plans, documents-sources, photographies anciennes et récentes, portraits.

Le parti pris du livre est " d'offrir une interprétation de la trajectoire lorientaise de 1666 à nos jours à travers celle de ses ports " (p. 10). En effet, depuis "l'invention " de Lorient, les destins du/des port-s et de la ville sont intimement liés, même si le projet initial ne comportait pas de fondation citadine. Ainsi, en août 1666, Denis Langlois, directeur de la première Compagnie des Indes, délimite-t-il dans la lande du Faouëdic, sur les terres de Rohan-Guéméné, paroisse de Ploemeur, le site du chantier de construction navale de la Compagnie, en face de Port-Louis, port effectivement retenu pour être son siège océanique. La situation évolue dès le début du XVIII e siècle, avec l'érection en paroisse du lieu-dit "Orient ", berceau de la future ville de Lorient qui dispose à partir de 1738 d'institutions municipales. Il faut en effet de la main-d'œuvre pour le port-arsenal, point central de la configuration urbaine sur le temps long, et la croissance démographique champignon - 3000 habitants en 1700, 7000 en 1730 et $15000 / 18000$ vers 1760 - impose des transformations. À titre de comparaison, l'agglomération de Lorient compte aujourd'hui près de 220000 habitants, ce qui la positionne au quatrième rang de la Bretagne historique, derrière Nantes, Rennes et Brest.

L'ouvrage est divisé en six grandes parties qui scandent l'histoire de la ville portuaire, "deux fois nouvelle à trois siècles d'intervalle " (p. 345) : "Le port et la ville de la Compagnie des Indes, des origines à 1770 " (p. 15-73); " De l'âge d'or lorientais à la fin d'un monde, 1770-1820 » (p. 75-122); « Lorient, quatrième port-arsenal français : innovation technologique et développement, 1820-1914 " (p. 123-190); " Du modèle portuaire idéal à la destruction de Lorient, 1914-1945 " (p. 191-232); "Reconstruction et nouvelles dynamiques portuaires, 1945-1992 (pages 233-298); Le temps des reconversions et des défis, 1993-2016 " (p. 299-344). Derrière cette chronologie émerge ce que Gérard Le Bouëdec qualifie de " trilogie portuaire lorientaise " : d'abord, le port des trois Compagnies des Indes successives et du commerce 
français avec l'Asie (XVII -XVIII ${ }^{e}$ siècles); ensuite, le port-arsenal de la Marine, dont les origines remontent au XVII ${ }^{\mathrm{e}}$ siècle mais dont l'essor intervient au cours du XIX ${ }^{\mathrm{e}}$ siècle, période marquée par l'avènement des bâtiments en fer et à vapeur, puis de gigantesques cuirassés; enfin, le port de la pêche hauturière, de l'invention du chalutage à vapeur comme alternative aux pêches côtières au tournant du $\mathrm{Xx}^{\mathrm{e}}$ siècle, jusqu'à l'armement Scapêche du groupe Intermarché. Ces transformations nécessitent des aménagements et une extension des installations portuaires depuis le noyau originel des chantiers de la Compagnie des Indes, investi par la Marine, avec la poldérisation des lagunes situées au nord de la rive droite du Scorff et sur la rive gauche, puis la création, toujours avec le soutien de la Marine, d'un quai pour le commerce à Kergroise, et d'un complexe portuaire complet pour la pêche à Keroman.

C'est peu dire que le rôle de l'État a été déterminant dans l'histoire de Lorient dès sa naissance sous le règne personnel de Louis XIV, au moment où Jean-Baptiste Colbert cherche à appliquer les doctrines mercantilistes à l'économie française. La présence et le développement d'infrastructures militaires constituent sur la longue durée le signe de la présence de la monarchie puis de la république. La période de l'occupation allemande pendant la Seconde Guerre mondiale voit Lorient devenir la plus grande base pour U-boote du Reich et conduit les Alliés à bombarder la ville et ses ports au début de l'année 1943, avec pour conséquence leur destruction totale. Si la reconstruction après le conflit s'est faite avec l'appui de l'État, les dernières décennies - à partir des années 1990 - sont caractérisées par un effacement de celui-ci, obligeant les pouvoirs publics lorientais à prendre la main sur le destin de la cité, avec un niveau décisionnel jamais atteint jusqu'alors. Les orientations prises montrent que Lorient continue à écrire son destin en regardant vers la mer.

En définitive, ce volume dédié au cas lorientais, relatant avec une grande minutie trois siècles et demi d'activités et de recompositions, comportant de précieux tableaux chiffrés et d'utiles index des noms de lieux (jusqu'aux quartiers et lieuxdits) et de personnes, s'impose comme un livre de référence pour quiconque s'intéresse à l'histoire maritime et portuaire.

Bernard Michon

LE BOUËDEC, Gérard, Lorient et le Morbihan. Une histoire de ressentiments et de rivalités (1666-1914), Rennes, Presses universitaires de Rennes, Collection Histoire, $2019,140 \mathrm{p}$.

Ce court essai s'inscrit dans la continuité de l'ouvrage que Gérard Le Bouëdec a publié avec Christophe Cérino, chez le même éditeur, sous le titre : Lorient, ville portuaire. Une nouvelle histoire des origines à nos jours. Il s'agit cette fois de "suivre l'onde de choc de ce séisme lorientais pour comprendre les réactions, les frustrations, les ressentiments qui nourrissent consciemment ou inconsciemment des rivalités durables " (p. 7-8). La création de Lorient en 1666 et l'implantation réussie de ce " corps étranger " sur un territoire qui devient au moment de la Révolution le Morbihan, provoque en effet de nombreux bouleversements, puisque cette ville nouvelle ne tarde pas à vouloir en dominer la partie occidentale, en attendant plus. C'est bien la question des pouvoirs et de leur éventuelle redistribution qui est au cœur des conflits avec Vannes, bien entendu, mais aussi avec Ploemeur - la paroisse d'implantation de Lorient -, Port-Louis ou Hennebont.

Pour construire son analyse, Gérard Le Bouëdec a pu s'appuyer sur ses propres recherches - en premier lieu sa thèse d'État, Le port et l'arsenal de Lorient, 